\title{
Low-Speed Aeroelastic Modeling of Very Flexible Slender Wings with Deformable Airfoils
}

\author{
Rafael Palacios ${ }^{1}$ \\ Imperial College, London SW7 2AZ, UK \\ Carlos E.S. Cesnik ${ }^{2}$ \\ University of Michigan, Ann Arbor, Michigan 48109-2140, USA
}

\begin{abstract}
A low-order aeroelastic model is introduced for very flexible high-aspect-ratio wings with adaptive airfoils. A geometrically-nonlinear beam-like model capable of capturing plate-like deformations has been coupled with a 2-D finite-state aerodynamic model with arbitrary airfoil deformations. The proposed approach results in a natural extension to the conventional way of analyzing high-aspect-ratio wings, with very little additional complexity. The control effectiveness of camber deformation is numerically investigated in some simple situations, including thin isotropic and anisotropic plates and a straight wing with a constant NACA4406 airfoil.
\end{abstract}

\section{Introduction}

$\mathrm{T}$ HIS paper investigates the effect of airfoil deformations in the aeroelastic response of slender wings with integral actuation. Different mechanisms that change airfoil camber have been proposed as an aerodynamically efficient substitute to discrete flap actuation to modify the aerodynamic forces, starting with Parker's (unsuccessful) variable-camber rib of 1920, which was presented as a high-lift mechanism for take-off and landing ${ }^{5}$. More recently proposed conformal shape changes for active aeroelastic pplications are mostly based in either the use of embedded smart actuators (typically piezoelectrics) in the wing structure ${ }^{1}$, or through compliant substructures, such as airfoils with deformable leading ${ }^{2}$ or trailing ${ }^{3}$ edges. Active twist rotors $^{1,4}$, in which the blade twist is controlled by embedded piezoelectric actuators, can be seen as the first generation of proposed morphing blades, although more complex shape changes have already started to be proposed ${ }^{6}$.

Typically, low-order aeroelastic analyses of both slender wings and rotorcraft blades are carried out using geometrically-nonlinear beam models for the structure, which assume that the cross sections are rigid. For low-speed flow, this structural model is complemented by unsteady airfoil theories (and an appropriate wake model) that provide the aerodynamic loading. However, there are situations in which the assumption of rigid cross sections cannot be justified, which can happen intentionally by design, as in the examples above $e^{1-5}$, or simply due to the

\footnotetext{
${ }^{1}$ Lecturer, Department of Aeronautics, 355 Roderic Hill Building (rpalacio@imperial.ac.uk). Member AIAA

${ }^{2}$ Associate Professor, Department of Aerospace Engineering, 3024 FXB Building (cesnik@umich.edu). Associate Fellow, AIAA
} 
compliance of the structure ${ }^{7-8}$. The approach to model morphing airfoils has been to represent the structure using plates or solid elements so as to include the airfoil deformations. On the aerodynamics side, models for deforming airfoils can include CFD and panel methods, or also lifting-line methods coupled with semi-empirical corrections. However, the presence of a dominant dimension makes 2-D models still attractive for a first estimation of the aeroelastic response. A potential-flow unsteady aerodynamics model for airfoils with arbitrary boundary conditions was developed by Sears ${ }^{9}$ for continuous gust response as an extension to Theodorsen's oscillating airfoil model ${ }^{10}$. General deformations of the airfoil were later introduced by $\mathrm{Wu}^{11,12}$ for the analysis of the swimming propulsion of fish, modeled as plate wave deformations of the form $z(x, t)=z_{0} \sin (\omega t-k x)$. A generalization of these solutions to transient responses was introduced by Peters ${ }^{13,14}$ by means of a finite-state solution procedure that decouples an inflow model of the unsteady wake effects from the calculation of loads. This later approach will be followed in this paper.

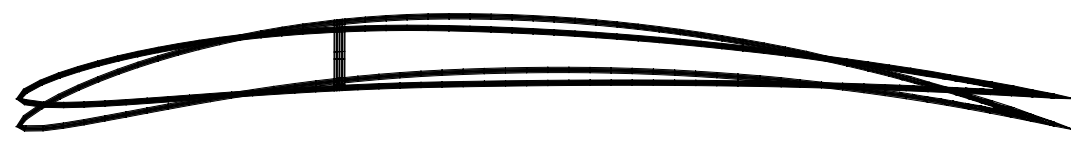

Figure 1. Camber deformation on a NACA4406 airfoil

This paper presents a procedure for the analysis of high-aspect-ratio wings with deformable airfoils that preserves the 1-D structural model and the 2-D aerodynamic model, but with additional degrees of freedom for the airfoil deformations. It is therefore based on a 1-D finite-element modeling of the structure, which now includes additional degrees of freedom to allow for sectional deformations. We have called finite-section deformation modes ${ }^{15}$ and they arise from a Ritz approximation to the local sectional warping field, such as those shown in Figure 1. The modal amplitudes are the additional degrees of freedom, with associated stiffness and inertia characteristics ${ }^{17}$. This structural model is used in conjunction with a 2-D unsteady aerodynamics model that also includes airfoil deformations through a Glauert expansion of the pressure and inflow-velocity fields ${ }^{14}$. A truncation of the series provides a finite-state approximation in time-domain of the aerodynamic equations, which are solved with the 1-D structural elements to provide the transient aeroelastic response of the system either to varying flight conditions (gust encounter, maneuvers, etc.) or to a geometric change induced by the embedded actuation.

The proposed approach results in a natural extension to the conventional way of analyzing slender wings, with very little additional complexity. A typical application of the present theory is the 1-D modeling of slender wings that can deform its camber (Figure 1). This might happen either directly from the pressure distribution due to the aerodynamic loading, or by an internal/embedded actuation mechanism and both situations are discussed in the paper. 


\section{Theoretical formulation}

\section{A. Structural model}

The structural model used in the present analysis has been described in Refs. 15 and 17. It follows the variationalasymptotic method for the analysis of composite beams ${ }^{16}$ : the equations of motion for a slender anisotropic elastic 3-D solid are approximated by the recursive solution of a linear 2-D problem at each cross section ${ }^{16}$, and a 1-D geometrically-nonlinear problem along the reference line ${ }^{18}$. This procedure allows the asymptotic approximation of the 3-D warping field in the beam cross sections, which are used with the 1-D beam solution to recover a 3-D displacement field. In a first development of the theory ${ }^{19}$, the warping was approximated for the elastic degrees of freedom of a Timoshenko-beam model (extension, twist, bending, and transverse shear). The present implementation adds an arbitrary expansion of the warping field through a set of functions approximating the sectional deformation field. It captures "non-classical" deformations, which are referred to as finite-section modes. And these new deformation modes are not restricted to be as small as small as the fundamental warping field. They can be introduced as follows: Let $\mathbf{X}$ be the position vector in the current configuration of the material point initially at $\left(x, x_{2}, x_{3}\right)$, with $x$ being the coordinate along the beam reference line and $x_{\alpha}$ the cross-sectional coordinates. Also let $\mathbf{B}_{\mathbf{i}}$ be the base vectors of a reference frame that moves with the solid (see Figure 2).

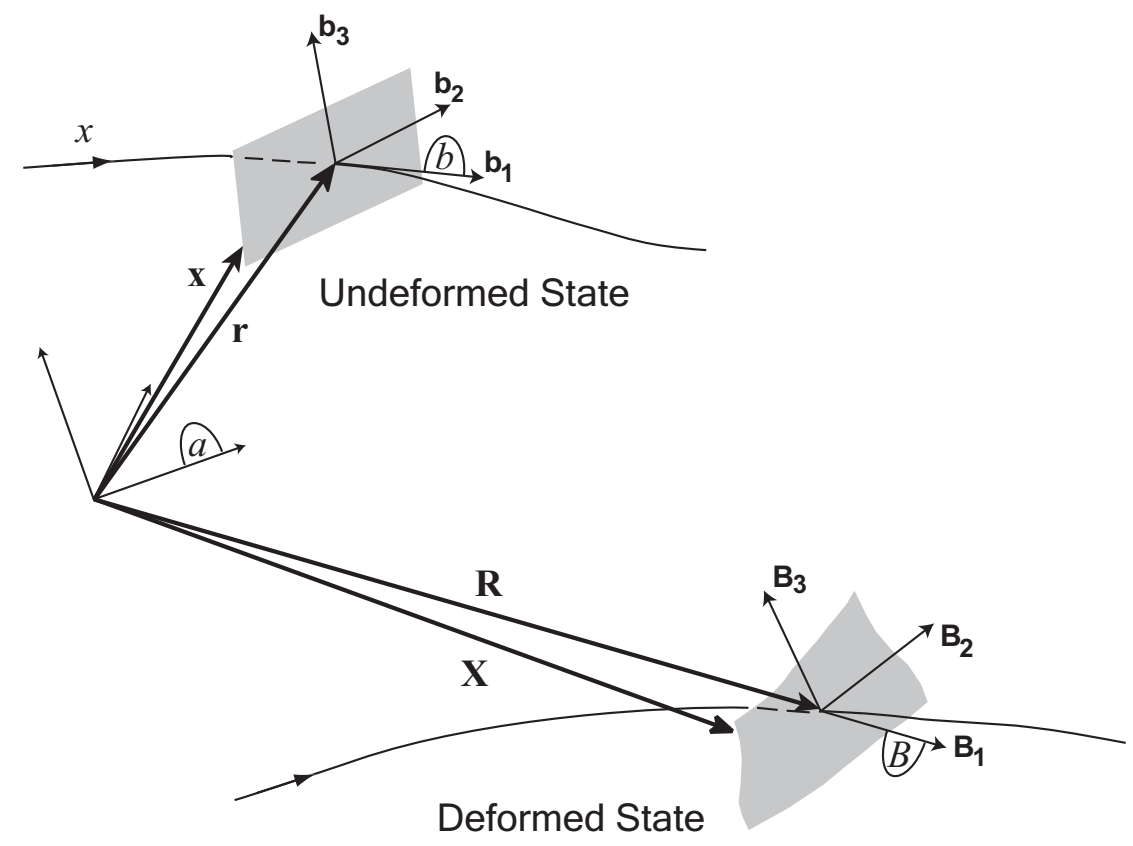

Figure 2. Undeformed and deformed states in the deformation

The position vector can be written, without loss of generality, as

$$
\mathbf{X}\left(x, x_{2}, x_{3}\right)=\mathbf{R}(x)+x_{\alpha} \mathbf{B}_{\boldsymbol{\alpha}}(x)+w_{i}\left(x, x_{2}, x_{3}\right) \mathbf{B}_{\mathbf{i}}(x)+\sum_{m=1}^{N_{q}} \Psi_{q_{m} i}\left(x_{2}, x_{3}\right) q_{m}(x) \mathbf{B}_{\mathbf{i}}(x),
$$


where $\Psi_{q_{m} i}$, with $m=1, \ldots, N_{q}$, is the set of prescribed finite-section modes, $q_{m}$ are the amplitudes of those modes, and $\mathbf{w}=w_{i} \mathbf{B}_{\mathbf{i}}$ is the residual warping displacement vector. For the description in Eq. (1) to be unique, the finitesection modes need to satisfy a set of orthogonality conditions ${ }^{17}$. In this expression, it is implied the summation on repeated indexes (ranging from 1 to 3 for Latin subindexes, and from 2 to 3 for Greek subindexes).

From the spatial differentiation of the displacement field (1) one can obtain the distribution of strains in the 3-D solution, which will be assumed to be small. In particular, we use the local Jaumann-Biot-Cauchy strain tensor ${ }^{20}$, which, for the displacement field given in Eq. (1), can be written as

$$
\boldsymbol{\Gamma}=\boldsymbol{\Gamma}_{\gamma_{1 i}} \gamma_{1 i}(x, t)+\boldsymbol{\Gamma}_{\mathbf{\kappa}_{i}} \kappa_{i}(x, t)+\sum_{m=1}^{N_{q}} \boldsymbol{\Gamma}_{\mathbf{q}_{\mathbf{m}}} q_{m}(x, t)+\sum_{m=1}^{N_{q}} \boldsymbol{\Gamma}_{\mathbf{q}_{\mathbf{m}}^{\prime}} q_{m}^{\prime}(x, t)+\boldsymbol{\Gamma}_{\mathbf{w}_{i}} w_{i}+\boldsymbol{\Gamma}_{\mathbf{l}_{i}} w_{i}^{\prime},
$$

where $\Gamma_{(\bullet)}$ are a set of tensor operators defined as in Cesnik and Hodges ${ }^{21}$, and $\gamma_{1 i}$ and $\kappa_{i}$ are the force and moment generalized strains, respectively, defined as

$$
\gamma=\left\{\begin{array}{l}
\gamma_{11} \\
2 \gamma_{12} \\
2 \gamma_{13}
\end{array}\right\}=C^{B a} R_{a}^{\prime}-C^{b a} r_{a}^{\prime}, \quad \kappa=\left\{\begin{array}{l}
\kappa_{1} \\
\kappa_{2} \\
\kappa_{3}
\end{array}\right\}=K_{B}-k_{b},
$$

where $C^{B a}$ and $C^{b a}$ are the rotation matrices between the deformed/undeformed frames and the moving frame, respectively, and $K_{B}$ and $k_{b}$ are the column vectors with the components of the curvature vectors of the deformed and undeformed frame, respectively. Subindexes are used to express the reference frame in which the component of the vector is projected. Analogously, the differentiation in time of the displacement field (1) defines the distribution of velocities in the solid ${ }^{15}$. In this case we neglect the contribution of the inertia associated with the warping (although not that of the finite-section modes). The local inertial velocity vector $\dot{\mathbf{X}}$ can be obtained as

$$
\dot{\mathbf{X}}=\mathbf{V}+\boldsymbol{\Omega} \times\left(x_{\alpha} \mathbf{B}_{\alpha}+\sum_{m=1}^{N_{q}} \Psi_{q_{m} i} q_{m} \mathbf{B}_{\mathbf{i}}\right)+\sum_{m=1}^{N_{q}} \Psi_{q_{m} i} \dot{q}_{m} \mathbf{B}_{\mathbf{i}}
$$

The components of the translational, $\mathbf{V}=V_{B i} \mathbf{B}_{\mathbf{i}}$, and rotational, $\mathbf{\Omega}=\Omega_{B i} \mathbf{B}_{\mathbf{i}}$, velocity vectors (defined with respect to the inertial reference frame) in Eq. (4) are given by

$$
\begin{gathered}
V_{B}=C^{B a}\left(\dot{R}_{a}+\tilde{\omega}_{a} R_{a}+v_{a}\right), \\
\tilde{\Omega}_{B}=-\dot{C}^{B a} C^{a B}+C^{B a} \tilde{\omega}_{a} C^{a B},
\end{gathered}
$$

where $v_{a}$ and $\omega_{a}$ are the translational and angular velocities of the moving reference frame (e.g., the hub frame in helicopter applications or the velocity of the center of gravity in maneuvering aircraft). The cross-sectional problem is set up by imposing the local minimization of the strain energy corresponding to the strain field, given by Eq. (2), for prescribed values of the 1-D generalized beam strains $\left\{\gamma, \kappa, q, q^{\prime}\right\}$, where $\gamma$ and $\kappa$ are the column matrices 
defined in (3) and $q=\left\{\begin{array}{lll}q_{1} & q_{2} & \ldots\end{array}\right\}^{T}$. The solution to this problem gives the warping field for given values of the 1D generalized beam strains. In its first order approximation, it can be written as ${ }^{17}$

$$
w\left(x_{1}, x_{2}, x_{3}\right)=w_{\gamma}\left(x_{2}, x_{3}\right) \gamma\left(x_{1}\right)+w_{\kappa}\left(x_{2}, x_{3}\right) \kappa\left(x_{1}\right)+w_{q}\left(x_{2}, x_{3}\right) q\left(x_{1}\right)+w_{q^{\prime}}\left(x_{2}, x_{3}\right) q^{\prime}\left(x_{1}\right)+\text { H.O.T. },
$$

where $\left\{w_{\gamma} w_{\kappa} w_{q} w_{q^{\prime}}\right\}$ are the first-order warping influence coefficients. Substitution of Eq. (6) into Eq. (2) and integration at the cross section gives the strain energy per unit length of the beam,

$$
\mathcal{U}=\frac{1}{2}\left\{\begin{array}{llll}
\gamma^{T} & \kappa^{T} & q^{T} & q^{\prime T}
\end{array}\right\}[S]\left\{\begin{array}{c}
\gamma \\
\kappa \\
q \\
q^{\prime}
\end{array}\right\}+\text { H.O.T. }
$$

The integration of the energy (7) is carried out by means of a finite-element discretization of the cross section. The constant matrix $[S]$ is the first-order asymptotic approximation to the stiffness matrix. The integration of the kinetic energy corresponding to the velocity field of Eq. (5) can be directly done as function of the 1-D variables, given by

$$
\mathcal{K}=\frac{1}{2}\left\{\begin{array}{lll}
V_{B}^{T} & \Omega_{B}^{T} & \dot{q}^{T}
\end{array}\right\}[M]\left\{\begin{array}{c}
V_{B} \\
\Omega_{B} \\
\dot{q}
\end{array}\right\},
$$

where the constant matrix $[M]$ is the inertia matrix for the cross section and negligible contribution of $\mathbf{\Omega} \times \boldsymbol{\Psi}_{\mathbf{q}_{\mathbf{m}}} q_{m}$ is assumed. If we include internal actuation loads into the problem (i.e., thermal forces or piezoelectric forces with prescribed voltage), the constitutive relations of the beam are finally written as

$$
\left\{\begin{array}{c}
F_{B} \\
M_{B} \\
Q_{S_{0}} \\
Q_{S_{1}}
\end{array}\right\}=[S]\left\{\begin{array}{c}
\gamma \\
\kappa \\
q \\
q^{\prime}
\end{array}\right\}-\left\{\begin{array}{c}
F^{(a)} \\
M^{(a)} \\
Q_{s_{0}}^{(a)} \\
Q_{S_{1}}^{(a)}
\end{array}\right\}, \text { and }\left\{\begin{array}{c}
P_{B} \\
H_{B} \\
Q_{t}
\end{array}\right\}=[M]\left\{\begin{array}{c}
V_{B} \\
\Omega_{B} \\
\dot{q}
\end{array}\right\}
$$

In this equation, the superindex $\bullet^{(a)}$ indicates actuation loads per unit length, $P_{B}$ and $H_{B}$ are the local translational and rotational inertial momenta, respectively, and $F_{B}$ and $M_{B}$ are the sectional forces and moments. They are all expressed in their components in a reference frame attached to the deformed beam reference line. $Q_{S_{0}}$ and $Q_{S_{1}}$ are the generalized forces and moments, respectively, corresponding to the finite-section modes, and $Q_{t}$ is the corresponding generalized momenta (all three magnitudes are column matrices of dimension $N$, the number of finite-section modes). The geometrically-nonlinear dynamic equations of equilibrium along the reference line (as presented in Ref. 15) are written as

$$
\left(\frac{d}{d t}+\tilde{\Omega}_{B}\right) P_{B}=\left(\frac{d}{d x}+\tilde{K}_{B}\right)\left(F_{B}-f_{1}\right)+f_{0}
$$




$$
\begin{gathered}
\left(\frac{d}{d t}+\tilde{\Omega}_{B}\right) H_{B}+\tilde{V}_{B} P_{B}=\left(\frac{d}{d x}+\tilde{K}_{B}\right)\left(M_{B}-m_{1}\right)+\left(\tilde{e}_{1}+\tilde{\gamma}\right) F_{B}+m_{0}, \\
\frac{d}{d t} Q_{t}=\frac{d}{d x}\left(Q_{s_{1}}-f_{s_{1}}\right)-\left(Q_{s_{0}}-f_{s_{0}}\right) .
\end{gathered}
$$

where $e_{1}^{T}=\left\{\begin{array}{lll}1 & 0 & 0\end{array}\right\}$. The first two equations imply equilibrium of forces and moments, where $f_{0}$ and $m_{0}$ are the conventional (zero-order) applied forces and moments, respectively, per unit length on the beam, while $f_{1}$ and $m_{1}$ are the first-order loads associated to the work needed to deform the cross-section. The last equation in (10) includes the set of equilibrium equations corresponding to the finite-section modes. $f_{s_{0}}$ and $f_{s_{1}}$ are the applied generalized forces and moments corresponding to the finite section modes. With the warping influence coefficients given by Eq. (6), the applied forces per unit length in Eq. (10) are obtained from the following integrals over the cross sections, $A(x)$,

$$
\begin{array}{ll}
f_{0}=\int_{A(x)} \mu_{B} d A, & f_{1}=\int_{A(x)} w_{\gamma}^{T} \mu_{B} d A, \\
f_{s_{0}}=\int_{A(x)}\left(\Psi_{q}^{T}+w_{q}^{T}\right) \mu_{B} d A, & f_{s_{1}}=\int_{A(x)} w_{q^{\prime}}^{T} \mu_{B} d A, \\
m_{0}=\int_{A(x)} \xi_{B} \mu_{B} d A, & m_{1}=\int_{A(x)} w_{\kappa}^{T} \mu_{B} d A .
\end{array}
$$

with $\mu_{B}$ and $\xi_{B}$ being the components of the applied (distributed) force vector, $\boldsymbol{\mu}\left(x, x_{2}, x_{3}\right)$, and $\boldsymbol{\xi}=\mathbf{X}-\mathbf{R}$ in the deformed reference, $B$, respectively. Finally, $\Psi_{q}\left(x_{2}, x_{3}\right)$ is a matrix whose columns are the finite-section modes in the problem, i.e., $\Psi_{q}\left(x_{2}, x_{3}\right)=\left\{\begin{array}{lll}\Psi_{q_{1}} & \Psi_{q_{2}} & \cdots\end{array}\right\}$.

\section{B. Aerodynamic model}

Aerodynamic loads are obtained from the 2-D finite-state formulation for flexible airfoils presented in Ref. 14. It is assumed that the airfoil has infinitesimal thickness and is initially located in $[-b,+b]$ along the $x_{2}$ axis, as shown in Figure 3. The air moves with velocity $\left(U_{0}, V_{0}\right)$ and with gradient $V_{1} x_{2} / b$ relative to the airfoil.

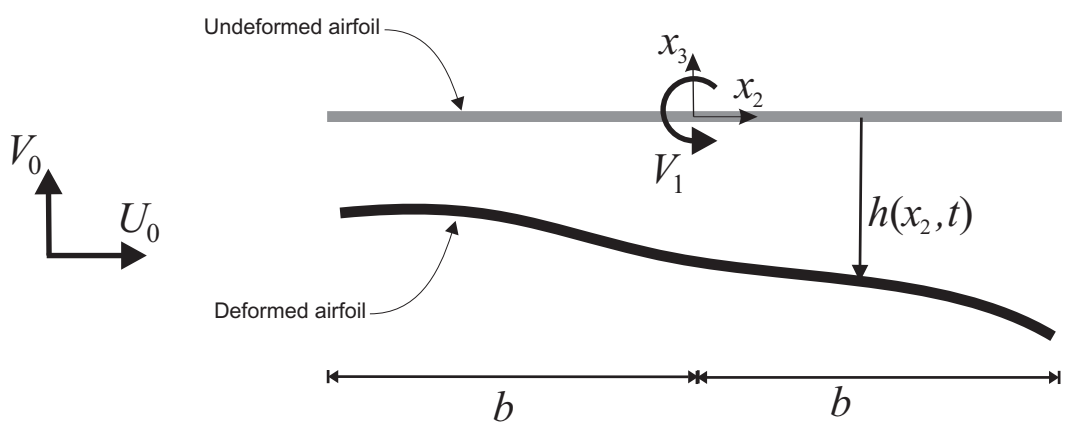




\section{Figure 3. Parameters used to represent a flexible airfoil}

The deformation of the airfoil is determined by $h\left(x_{2}\right)$, defined positive down as in Figure 3. It is assumed that the deformations within the reference frame are small, that is, $h<<b, \partial h / \partial x_{2}<<1$ and $\partial h / \partial t<<u_{0}$. The frame itself is allowed to have arbitrarily large motion although it will be assumed that the trailing vorticity is always located along the $y$ axis. The non-penetration boundary condition is expressed as

$$
v=\bar{v}+\lambda=U_{0} \frac{\partial h}{\partial x_{2}}+\frac{\partial h}{\partial t}+V_{0}+V_{1} \frac{x_{2}}{b},
$$

where $v$ is the total induced vertical velocity, $\lambda$ is the induced flow from wake vorticity, and $\bar{v}$ is the induced flow from bound circulation. The solution to the integral equations that give the aerodynamic forces is found using Glauert's procedure of expanding the vorticity in Chebyshev polynomials ${ }^{23}$. For the case of arbitrary airfoil displacements, the deformation of the flexible airfoil will be also approximated by such expansion, as ${ }^{14}$

$$
h(\xi)=\sum_{n=0}^{\infty} h_{n} T_{n}(\xi), \quad \text { with } \quad h_{n}=\frac{\mu_{n}}{\pi} \int_{-1}^{1} h(\xi) \frac{T_{n}(\xi)}{\sqrt{1-\xi^{2}}} d \xi \quad \text { and } \quad \mu_{n}= \begin{cases}1, & \text { if } n=0 \\ 2, & \text { if } n>0\end{cases}
$$

where $T_{n}(\xi)$ are the Chebyshev polynomials of the first $\operatorname{kind}^{22}$ along the non-dimensional chordwise direction, $\xi=\frac{x_{2}}{b} \in[-1,1]$. Substituting Eq. (13) into (12) we obtain the following expression for the airfoil velocity:

$$
v(\xi)=\sum_{n=0}^{\infty} v_{n} T_{n}(\xi)=V_{0}+V_{1} \xi+\sum_{n=0}^{\infty}\left[\dot{h}_{n}+\frac{U_{0} \mu_{n}}{b} \sum_{m=n+1, n+3}^{\infty} m h_{m}\right] T_{n}(\xi)
$$

The pressure increase on the airfoil, $\Delta P$, corresponding to this induced velocity can now be calculated and integrated along the chord. The following generalized forces are defined

$$
L_{n}=-b \int_{-1}^{1} T_{n}(\xi) \Delta P d \xi .
$$

which are given by ${ }^{14}$

$$
\begin{aligned}
& L_{0}=\frac{2 \pi \rho b}{\mu_{0}}\left[U_{0}\left(-\frac{v_{0}-\lambda_{0}}{\mu_{0}}-\frac{v_{1}}{\mu_{1}}\right)-\frac{b}{2}\left(\frac{\dot{v}_{0}}{\mu_{0}}-\frac{\dot{v}_{2}}{\mu_{2}}\right)\right], \\
& L_{1}=\frac{2 \pi \rho b}{\mu_{1}}\left[U_{0}\left(\frac{v_{0}-\lambda_{0}}{\mu_{0}}-\frac{v_{2}}{\mu_{2}}\right)-\frac{b}{4}\left(\frac{\dot{v}_{1}}{\mu_{1}}-\frac{\dot{v}_{3}}{\mu_{3}}\right)\right] \\
& L_{n}=\frac{2 \pi \rho b}{\mu_{n}}\left[U_{0}\left(\frac{v_{n-1}}{\mu_{n-1}}-\frac{v_{n+1}}{\mu_{n+1}}\right)+\frac{b}{2(n-1)}\left(\frac{\dot{v}_{n-2}}{\mu_{n-2}}-\frac{\dot{v}_{n}}{\mu_{n}}\right)-\frac{b}{2(n+1)}\left(\frac{\dot{v}_{n}}{\mu_{n}}-\frac{\dot{v}_{n+2}}{\mu_{n+2}}\right)\right] \text { for } n \geq 2 .
\end{aligned}
$$

It is interesting to note that the first two generalized loads, which correspond to the lift and the moment about the midchord, are completely defined by the first three terms of the velocity expansion, that is, plunge, pitch, and camber of the airfoil. The generalized forces also depend on $\lambda_{0}$, defined as the zero-order coefficient in the expansion of the inflow $\lambda$, 


$$
\lambda(\xi)=\sum_{n=0}^{\infty} \lambda_{n} T_{n}(\xi), \text { with } \lambda_{n}=\frac{\mu_{n}}{\pi} \int_{-1}^{1} \frac{T_{n}(\xi)}{\sqrt{1-\xi^{2}}} \lambda(\xi) d \xi
$$

As it was mentioned above, a 2-D flat wake is assumed, for which simple finite-state model can be obtained ${ }^{23}$. The coefficients of the expansion of the inflow in Eq. (17) are then determined by the following differential equations

$$
\frac{b}{n}\left(\frac{\dot{\lambda}_{n-1}}{\mu_{n-1}}-\frac{\dot{\lambda}_{n+1}}{\mu_{n+1}}\right)+U_{0} \lambda_{n}=\frac{\dot{\Gamma}}{n \pi}, \quad \text { with } n \geq 1
$$

with the bound circulation, $\Gamma$, given by

$$
\Gamma=2 \pi b\left[\left(v_{0}-\lambda_{0}\right)+\frac{1}{2}\left(v_{1}-\lambda_{1}\right)\right] .
$$

To close the system, an approximation for $\lambda_{0}$ is also needed, and it is provided by the expression ${ }^{23}$

$$
\lambda_{0} \approx \frac{1}{2} \sum_{n=1}^{\infty} \frac{(N+n) !}{(N-n) !} \frac{(-1)^{n-1}}{(n !)^{2}} \lambda_{n}
$$

\section{Coupled aeroelastic equations}

Eq. (11) has introduced the definition of the generalized forcing terms on a 1-D model of a structure with sectional deformations. They can be obtained from the expansion of the aerodynamic forces defined in Eq. (16). For that purpose, we first need to compute the corresponding expansion in Chebyshev polynomials of the thickness-averaged sectional deformations. For each one of the finite-section modes, $\Psi_{q_{m}}\left(x_{2}, x_{3}\right)$, defined as assumed displacements over the cross section, we define the following expansion,

$$
\left\langle\Psi_{q_{m} 3}\right\rangle \equiv \frac{\int \Psi_{q_{m} 3} d x_{3}}{\int d x_{3}}=\sum_{n=0}^{\infty} \psi_{q_{m} n} T_{n}(\xi), \quad \text { with } \quad \psi_{q_{m} n}=\frac{\mu_{n}}{\pi} \int_{-1}^{1}\left\langle\Psi_{q_{m} 3}\right\rangle \frac{T_{n}(\xi)}{\sqrt{1-\xi^{2}}} d \xi
$$

where $\Psi_{q_{m} 3}\left(x_{2}, x_{3}\right)$ refers to the component along the vertical direction of the $m$-th finite-section mode. The forcing terms corresponding to the finite-section modes for Eq. (10) can be obtained from the generalized aerodynamic forces (16) by the relation

$$
f_{s 0_{m}}=-\sum_{n=0}^{\infty} \psi_{q_{m} n} L_{n}
$$

with $m=1, \ldots, N_{q}$, the number of finite-section modes introduced in the analysis. Similar expressions are obtained to include the contributions of the warping influence coefficients introduced in Eq. (6), and corresponding to different beam deformation measures ${ }^{17}$.

The integration of the differential equations in the structural and inflow degrees of freedom is performed simultaneously in this work, using a simple explicit method system with iterative refinement to achieve the desired 
convergence. A mixed-form solution of the structural dynamics equations is used ${ }^{15}$, which solves simultaneously the equation in displacements, forces and momenta (this is particularly relevant for the recovery of the threedimensional displacement field from Eqs. (1) and (6), which depends on the 1-D internal forces). A finite-element discretization with constant-valued elements is used for the spatial integration of the equation, while a three-point backwards Euler scheme is used for the integration of the resulting equations in the time variable. The resulting nonlinear system is solved, along with the boundary conditions, by a Newton-Raphson scheme, with the Jacobians available in closed form. The solution of the linear inflow equations (18) on each airfoil is updated in each NewtonRaphson iteration such that convergence of the aerodynamics and structural equations is simultaneous within the time step.

\section{Harmonic oscillations of a thin airfoil with time-varying camber deformations}

The expressions for lift and moment created on a plate with arbitrary harmonic motions were already obtained by Sears ${ }^{9}$. Here we will investigate the problem of a plate with parabolic camber deformation shown in Figure 4, for which we also want to know the generalized load (bimoment) associated to the camber deformations. The three degrees of freedom are then the plunge motion, $\zeta$, the angle of attack, $\alpha$, and the amplitude of the camber deformation, $\delta$. It is assumed that all three of them are very small. In that case, the distribution of vertical displacemens along the airfoil is given by

$$
h\left(x_{2}, t\right)=\zeta+x_{2} \alpha+\delta\left[\left(\frac{x_{2}}{b}\right)^{2}-\frac{1}{3}\right] .
$$

The non-zero coefficients of the Chebyshev expansion of Eq. (23) are $\left\{h_{0}, h_{1}, h_{2}\right\}=\left\{\zeta+\frac{1}{6} \delta, b \alpha, \frac{1}{2} \delta\right\}$.

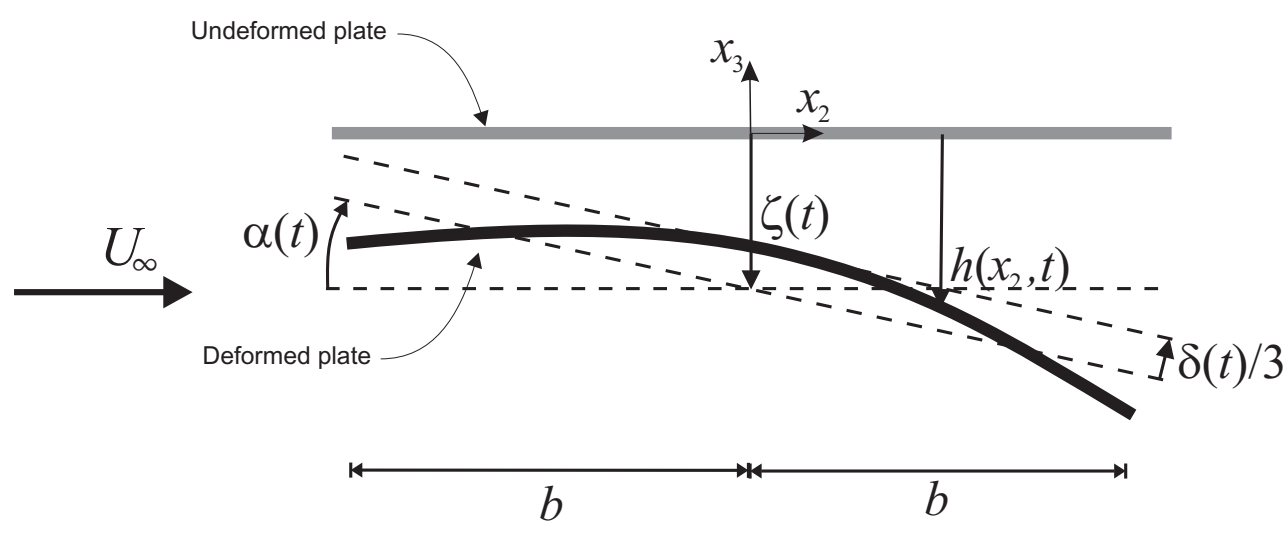

Figure 4. Thin-plate with camber deformation

The non-zero coefficients in the expansion of the total velocity on the airfoil, Eq. (14), can now be obtained as

$$
v_{0}=\dot{\zeta}+U_{\infty} \alpha+\frac{1}{6} \dot{\delta}, \quad v_{1}=b \dot{\alpha}+\frac{2 U_{\infty}}{b} \delta, \quad v_{2}=\frac{1}{2} \dot{\delta} .
$$


It will be further assumed that the plate has a flat wake and it moves with harmonic oscillations, that is, $\zeta=\bar{\zeta} e^{i \omega t}$, $\alpha=\bar{\alpha} e^{i \omega t}$, and $\delta=\bar{\delta} e^{i \omega t}$. In such case, the inflow component $\lambda_{0}$ is related to the Theodorsen function, $C(k)$, by the following relation ${ }^{14}$

$$
\lambda_{0}=\left(v_{0}+\frac{v_{1}}{2}\right)(1-C(k))
$$

where $k=\omega b / U_{\infty}$ is the reduced frequency of the oscillations. Substituting now Eq. (24) into Eq. (16) we find the following terms in the Glauert expansion of the pressure forces,

$$
\begin{gathered}
\frac{L_{0}}{\pi \rho b}=-2 U_{\infty} C(k)\left(\dot{\zeta}+U_{\infty} \alpha+\frac{b}{2} \dot{\alpha}+\frac{U_{\infty}}{b} \delta+\frac{1}{6} \dot{\delta}\right)-b\left(\ddot{\zeta}+U_{\infty} \dot{\alpha}-\frac{1}{12} \ddot{\delta}\right), \\
\frac{L_{1}}{\pi \rho b}=U_{\infty} C(k)\left(\dot{\zeta}+U_{\infty} \alpha+\frac{b}{2} \dot{\alpha}+\frac{U_{\infty}}{b} \delta+\frac{1}{6} \dot{\delta}\right)-U_{\infty}\left(\frac{b}{2} \dot{\alpha}+\frac{U_{\infty}}{b} \delta+\frac{1}{2} \dot{\delta}\right)-\frac{1}{8} b^{2} \ddot{\alpha} \\
\frac{L_{2}}{\pi \rho b}=\frac{b}{2} \ddot{\zeta}+U_{\infty} b \dot{\alpha}+\frac{U_{\infty}^{2}}{b} \delta-\frac{b}{12} \ddot{\delta} .
\end{gathered}
$$

This expressions can be also obtained as a particular case of Sears' solution ${ }^{9}$ for the induced velocity given by Eqs. (24). The aerodynamic loads corresponding to the three degrees of freedom defined in Eqs. (23) will be the lift per unit length, $L$, the moment per unit length about the half-chord point, $M_{1 / 2}$, and the camber bimoment, $\Lambda$ (which has dimensions of force per unit length according to the definition of $\delta$ ). They are obtained from Eq. (15), as

$$
\begin{aligned}
& L=\int_{-b}^{b} \Delta P d x_{2}=-L_{0}, \\
& M_{1 / 2}=\int_{-b}^{b} x_{2} \Delta P d x_{2}=b L_{1}, \\
& \Lambda=-\int_{-b}^{b}\left(\frac{x_{2}^{2}}{b^{2}}-\frac{1}{3}\right) \Delta P d x_{2}=\frac{1}{2} L_{2}+\frac{1}{6} L_{0} .
\end{aligned}
$$

The definition of $\Lambda$ implies that a positive camber bimoment produces a positive value of the camber measure $\delta$ defined in Figure 4. Finally, the static aerodynamic loads are obtained by neglecting the time derivatives in Eqs. (28). This results in the following static aerodynamic forces per unit span length,

$$
\begin{aligned}
& L_{s s}=2 \pi \rho U_{\infty}^{2} b\left(\alpha_{s s}+\delta_{s s} / b\right), \\
& M_{1 / 2, s s}=\pi \rho U_{\infty}^{2} b^{2} \alpha_{s s}, \\
& \Lambda_{s s}=\frac{1}{6} \pi \rho U_{\infty}^{2} b\left(\delta_{s s} / b-2 \alpha_{s s}\right) .
\end{aligned}
$$

The generalized aerodynamic forces corresponding to harmonic oscillations in pitch and, $\alpha$, camber $\delta$, were computed from Eqs. (26)-(28) and are compared in Figure 5 to Figure 6 with the numerical integration of the finitestate aerodynamics given by Eqs. (16) to (20). The non-dimensional coefficients in the figures are defined as $c_{l k}=L_{k} /\left(\rho U_{\infty}^{2} b\right)$. Eight inflow states were used and provide a very good approximation of the unsteady terms. 

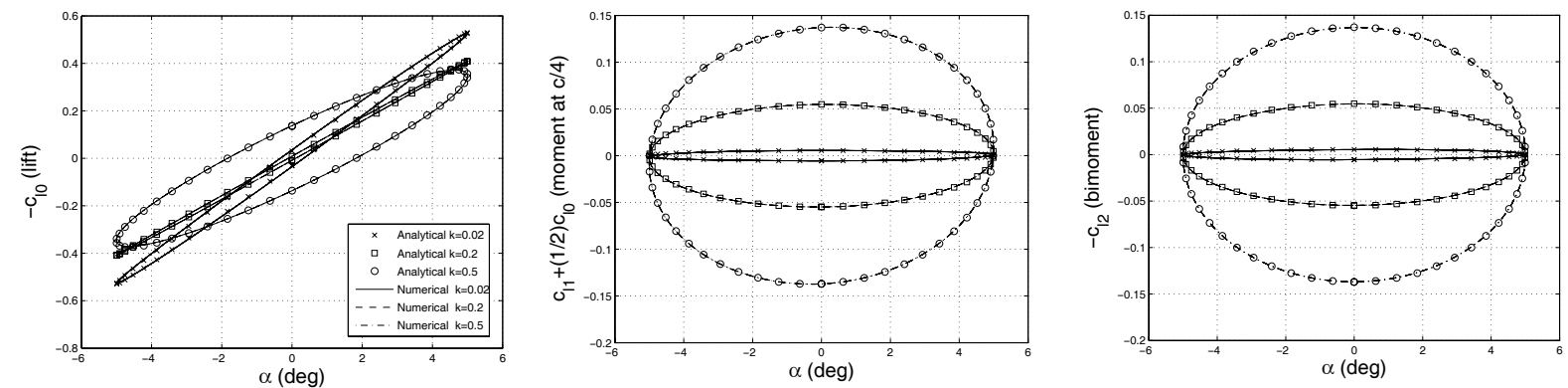

Figure 5. Generalized force coefficients on a flat plate with harmonic pitch motion
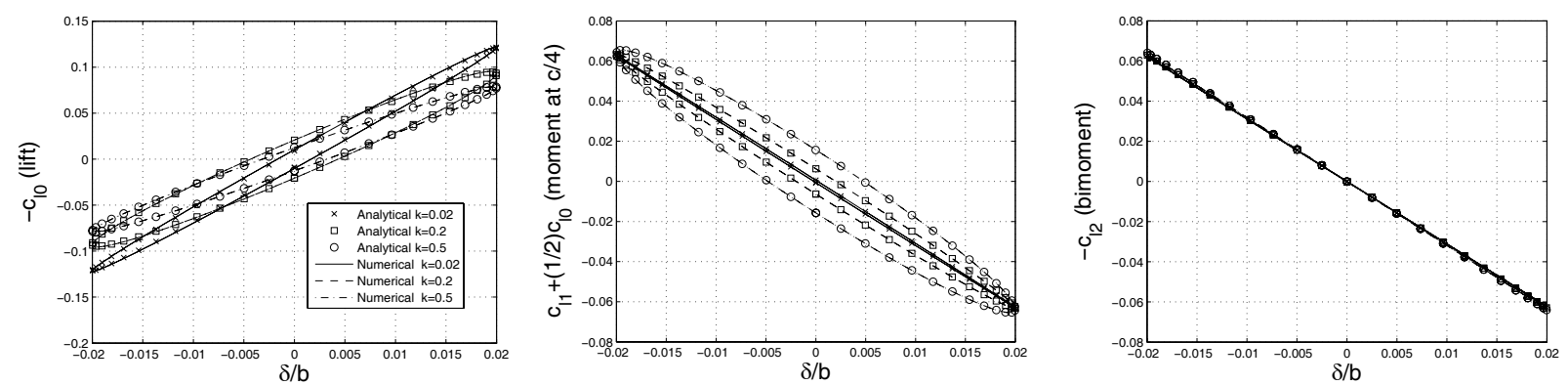

Figure 6. Generalized force coefficients on a flat plate with harmonic camber motion

Note that from Eqs. (26)-(28), for pure harmonic pitch oscillations, i.e., $\zeta=0, \alpha=\bar{\alpha} e^{i \omega t}$, and $\delta=0$, it is then $L_{1}+\frac{1}{2} L_{0}+L_{2}=-\frac{1}{8} \pi \rho b^{3} \ddot{\alpha}$, which is a rather small contribution for the reduced frequencies of interest in this work. This explains the very small differences between the aerodynamic coefficients for bimoment and moment about the 1/4-chord in Figure 5.

\section{Aeroelastic Test Cases}

The control effectiveness of camber deformation is numerically investigated in what follows for some simple configurations. They include a thin isotropic plate, a composite plate with surfaced-mounted piezoactuators, and a straight wing with NACA4406 airfoil. Airfoil deformations are limited in all cases to change of camber, which will be defined by a single finite-section mode in the structural model, as in Eq. (1). The prescribed finite-section mode will be the same as above, Eq. (23), and is given by

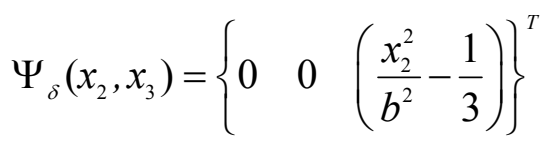

This particular parabolic mode does not change the position of the area centroid of the cross section, which is a basic requisite on the structural mode ${ }^{17}$. The structural deformations will be then characterized by the evolution of five elastic degrees of freedom along a reference line. They correspond to the extension, twist, and bending about all three axes. In the aerodynamic model, the airfoil deformations are defined by 6 to 8 terms of the Glauert expansion 
(13). This is to account for the warping of the cross section, Eq. (6), since only three terms would be needed to exactly capture the prescribed shape of Eq. (31). Although we are focusing here on camber bending deformations, it should be noticed that the procedure introduced in this work can accommodate any arbitrary sectional deformations in both the structural and the aerodynamic model.

\section{A. Isotropic flat plate}

The first numerical investigation on the effect of camber-bending deformations is carried out on an Aluminum flat plate (Young's modulus $E=72.4 \mathrm{GPa}$, Poisson's ratio $v=0.3$, and material density $\rho=2770 \mathrm{~kg} / \mathrm{m}^{3}$ ). Beam length is $\mathrm{L}=2 \mathrm{~m}$, the semichord is $b=0.1 \mathrm{~m}$ and the plate thickness is $t=6 \mathrm{~mm}$. The first to observe is the difference between the prescribed finite-section mode that has been selected, $\Psi_{\delta}$, given by Eq. (31), and the final warping field corresponding to the additional degree of freedom, i.e., $\Psi_{\delta}+w_{\delta}$. The warping influence coefficient $w_{\delta}$ was introduced in Eq. (6) after the evaluation of the local minimum of the cross-sectional strain energy about a mean value defined by the prescribed function $\Psi_{\delta}$ (see Ref. 8 for more details). Figure 7 compares the sectional deformations for $\delta / b=1$ obtained from Eq. (1) with and without sectional warping effects. The warping influence coefficients $w_{\delta}$ are a function of the geometry and the material distribution of the cross section, and, as shown in the figure, introduce a small (although far from negligible) correction in the final deformation shape of the isotropic plate.

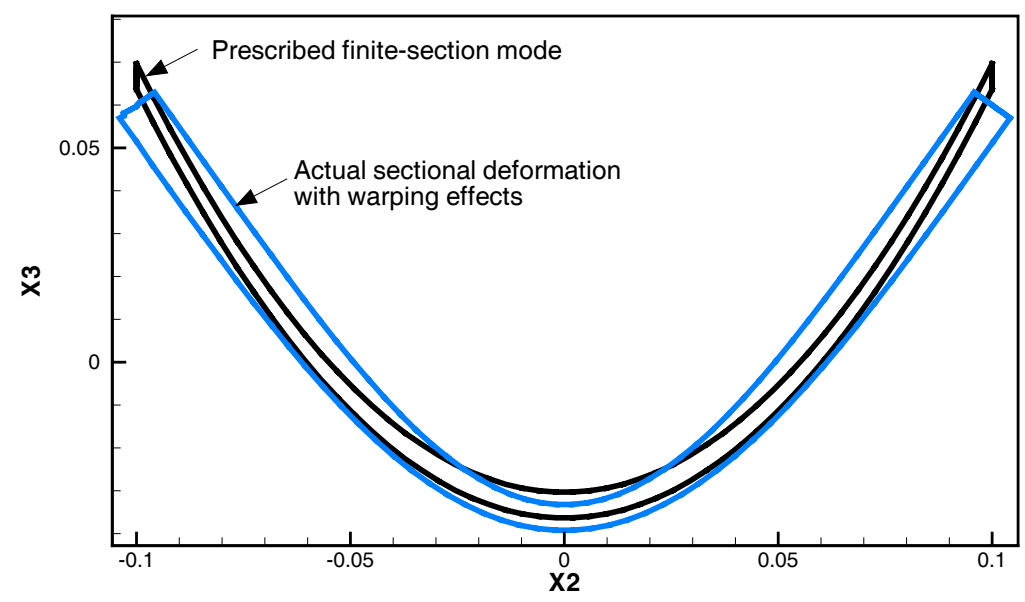

Figure 7. Effect of the warping field on an isotropic thin-plate with camber deformation $(\delta / b=1)$

The warping is computed on a finite-element discretization of the cross section, from which numerical integration provides the coefficients of the Glauert expansion of the airfoil deformations, according to Eq. (13). In this case, a good approximation was obtained with $N=8$ in the Glauert expansion and 50 elements along $x_{2}$ in the finiteelement model. The stiffness constant associated to an arbitrary finite-section mode, $\Psi_{q}$, on a homogenous isotropic cross section is given by ${ }^{24}$ 


$$
S_{q q}=\frac{1}{2} \lambda\left\langle\bar{w}_{i, i} \bar{w}_{j, j}\right\rangle+\mu\left\langle\bar{w}_{i, j} \bar{w}_{i, j}\right\rangle
$$

with $\bar{w}=\Psi_{q}+w_{q}$ and with $\lambda$ and $\mu$ Lamé constants of the material. For the particular case of camber-bending deformation, defined by the finite-section mode defined in Eq. (31), and if the warping field is neglected, Eq. (32) gives the following approximation to camber-bending stiffness

$$
\left.S_{\delta \delta}\right|_{\text {nowarp }}=\frac{8 \mu t}{3 b} .
$$

The steady-state forces in Eq. (30) provide now an estimation of the static camber deformation of an isotropic plate of thickness $t$ and semichord $b$, set at a given static angle of attack, $\alpha_{s s}$, with respect to a uniform flow of velocity $U_{\infty}$ and density $\rho$ :

$$
\frac{\delta_{s s}}{b} \approx-\frac{2}{16 \frac{\mu t}{\pi \rho U_{\infty}^{2} b}-1} \alpha_{s s} .
$$

As one could expect, camber deformations will be in general relatively small except for very thin plates and the compliance of the structure in this degree of freedom can be therefore neglected. Note also that equation (34) defines a divergence situation driven by the camber deformation at $U_{D}=4 \sqrt{(\mu t) /(\pi \rho b)}$. This velocity will be however very large in most practical situations.

From the estimation above, the compliance in camber of thin-plates for low-speed aerodynamic loads is typically unimportant, but it can however be quite relevant in morphing situations, that is, when the airfoil deformation is enforced by some internal mechanism. The effect of such mechanisms on the camber degree of freedom can in general be modeled as an applied bimoment on the corresponding sections. To illustrate this situation, we investigate the ability of the applied bimoments to stability low-damped oscillations in the $2 \times 0.2-\mathrm{m}$ Aluminum plate under consideration. Figure 8 shows the vertical shear force at the wing root on a cantilever plate at 5-deg angle of attack with respect to a sea-level $25-\mathrm{m} / \mathrm{s}$ uniform airstream. After an initial perturbation (of 1-deg amplitude over $\pi / 10$ seconds) the plate shows very-lightly damped oscillations, which were removed by appropriately tuning an applied point bimoment at the $70 \%$ section of the plate (the actual mechanism to apply that load is not discussed). This bimoment is activated at the instant $t=4 \mathrm{~s}$. The amplitude of camber deformation needed at the $70 \%$ section was $\delta=4.79 \%$ of the chord (with $\delta$ defined as in Figure 4), which was obtained with a bimoment $\Lambda=21 \mathrm{kN}$. The instantaneous deformed shape of the plate at $\mathrm{t}=8 \mathrm{~s}$, which is a point near a maximum of camber deformation, is shown in Figure 9. 


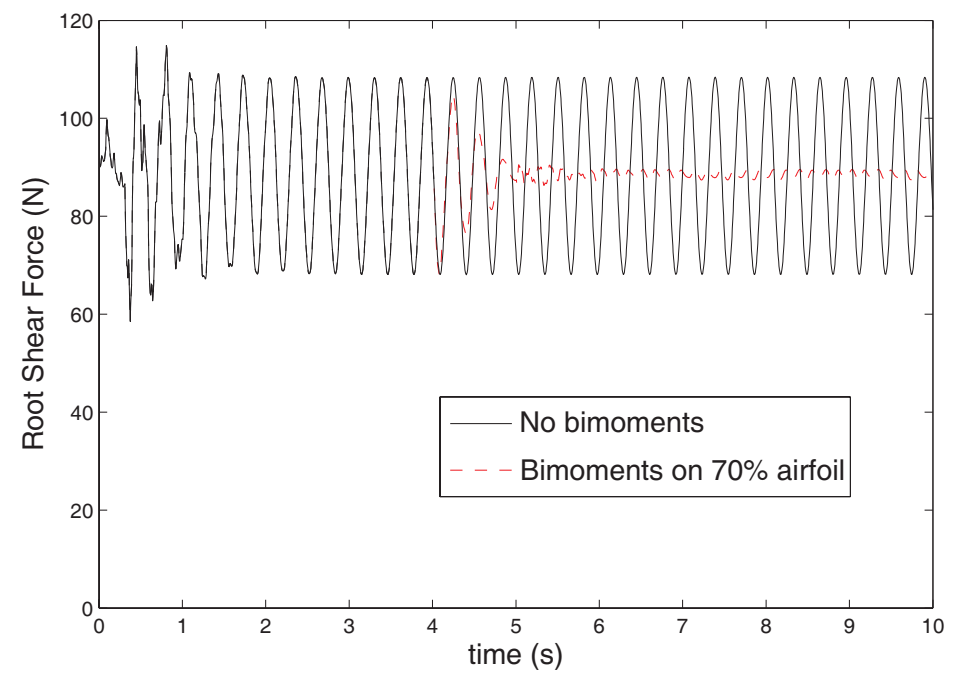

Figure 8. Cancellation of undamped root forces at $\mathrm{U}=\mathbf{2 5} \mathrm{m} / \mathrm{s}$ by using with camber bimoments

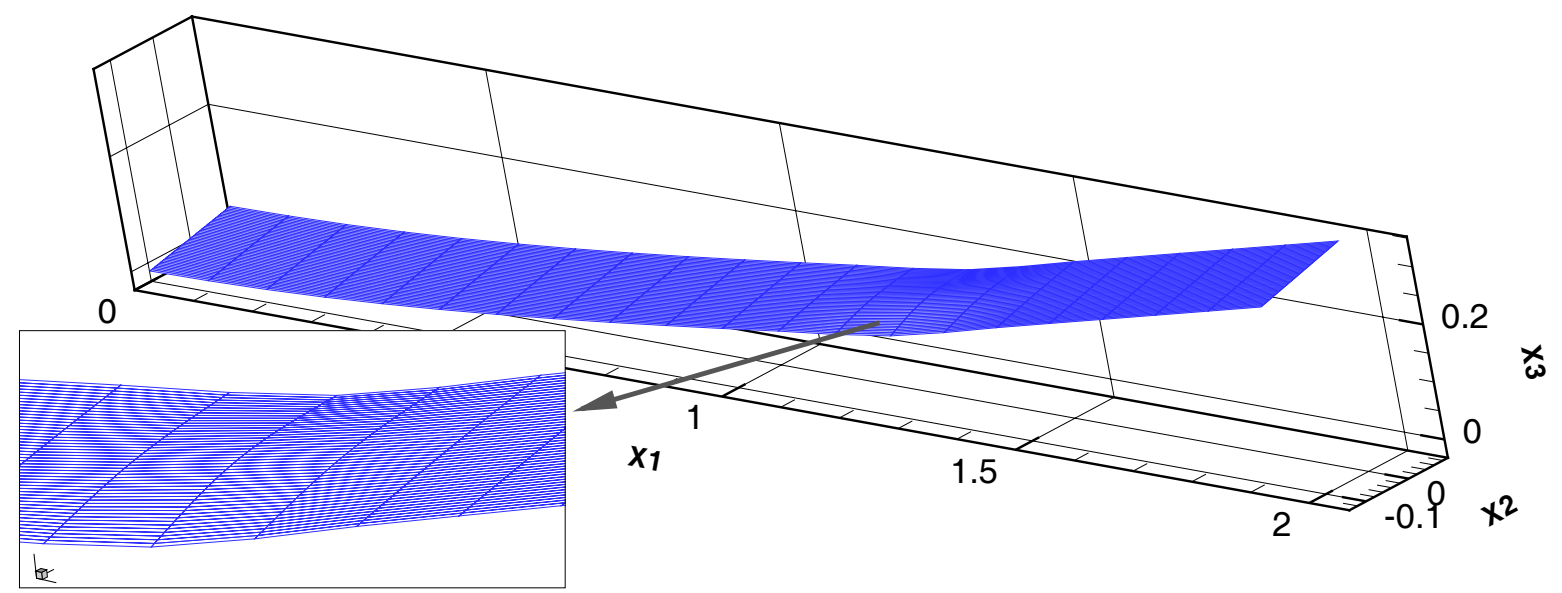

Figure 9. Instantaneous deformation of plate upper surface at $\mathrm{t}=\mathbf{8}$ sec (zoom: area where bimoments are applied)

\section{B. Composite flat plate with piezo actuation}

The second configuration is a graphite-epoxy thin strip with constant ply angle $(\phi)$ with respect to the longitudinal dimension (i.e., it will exhibit bending-torsion coupling for $\phi \neq 0 \mathrm{deg}$ and $\phi \neq 90 \mathrm{deg}$ ) and two surface-mounted piezoelectric (PZT-5H) actuators symmetrically bonded to it. The layout is shown in Figure 10. The elastic and dielectric constants for this case are those used in Ref. 24, where the static response of this configuration to PZT actuation was investigated in vacuum. For the present numerical analysis, the geometric parameters are $a=b=100$ $\mathrm{mm} ; c=0 ; h=20 \mathrm{~mm} ; t=1 \mathrm{~mm} ; t_{P Z T}=0.254 \mathrm{~mm}$. Static aeroelastic results were computed on this configuration for a constant voltage on the PZT actuators of $V=150 \mathrm{~V}$ (corresponding to a free strain of $240 \mu \varepsilon$ ). 


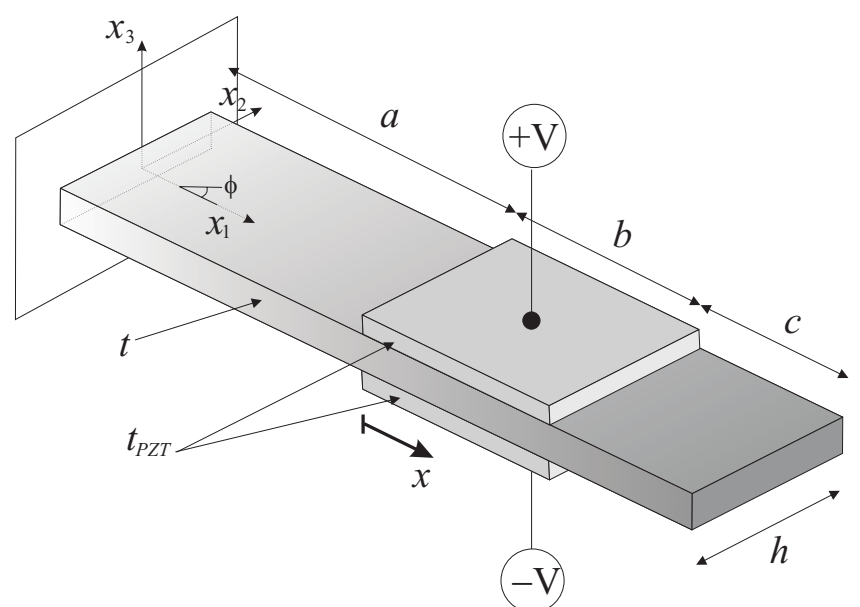

Figure 10. Composite thin strip with PZT actuators

Results are presented in Table 1 for a classical beam model (extension-twist-bending degrees of freedom) and for a 1-D structural model that also includes camber bending through a finite-section mode defined as in Eq. (31). Results are computed for the structure in vacuum and with airspeed of $U_{\infty}=25 \mathrm{~m} / \mathrm{s}$ at sea level. Table 1 shows the tip vertical displacements $\left(u_{3}\right)$, twist rotations $\left(\theta_{1}\right)$ and amplitude of camber deformations $(\delta)$ for a model with the reference line at the centerline of the strip. Three different ply angles, $\phi$, were studied.

Table 1. Tip displacements, twist rotation and amplitude of camber deformation

\begin{tabular}{|c|c|c|c|c|}
\hline & \multicolumn{4}{|c|}{$\phi=0 \mathrm{deg}$} \\
\hline & \multicolumn{2}{|c|}{$U_{\infty}=0$} & \multicolumn{2}{|c|}{$U_{\infty}=25 \mathrm{~m} / \mathrm{s}$} \\
\hline & w/o camber & with camber & w/o camber & with camber \\
\hline \multirow{6}{*}{$\begin{array}{c}\mathrm{u}_{3}(\mathrm{~mm}) \\
\theta_{1}(\mathrm{deg}) \\
\delta(\mathrm{mm}) \\
\end{array}$} & -1.71 & -1.70 & -1.71 & -1.58 \\
\hline & 0. & 0. & 0. & 0. \\
\hline & - & $-4.03 \times 10^{-2}$ & - & $-4.03 \times 10^{-2}$ \\
\hline & \multicolumn{4}{|c|}{$\phi=15 \mathrm{deg}$} \\
\hline & \multicolumn{2}{|c|}{$U_{\infty}=0$} & \multicolumn{2}{|c|}{$U_{\infty}=25 \mathrm{~m} / \mathrm{s}$} \\
\hline & w/o camber & with camber & w/o camber & with camber \\
\hline $\mathrm{u}_{3}(\mathrm{~mm})$ & -1.88 & -3.69 & -1.55 & -2.65 \\
\hline$\theta_{1}(\mathrm{deg})$ & 0.66 & 1.36 & 0.54 & 0.97 \\
\hline \multirow[t]{4}{*}{$\delta(\mathrm{mm})$} & - & $-7.74 \times 10^{-2}$ & - & $-7.74 \times 10^{-2}$ \\
\hline & \multicolumn{4}{|c|}{$\phi=30 \mathrm{deg}$} \\
\hline & \multicolumn{2}{|c|}{$\mathrm{U}_{\infty}=\mathbf{0}$} & \multicolumn{2}{|c|}{$U_{\infty}=25 \mathrm{~m} / \mathrm{s}$} \\
\hline & w/o camber & with camber & w/o camber & with camber \\
\hline $\mathrm{u}_{3}(\mathrm{~mm})$ & -2.30 & -4.46 & -1.17 & -1.36 \\
\hline$\theta_{1}(\mathrm{deg})$ & 1.14 & 2.40 & 0.87 & 1.65 \\
\hline$\delta(\mathrm{mm})$ & - & $-6.89 \times 10^{-2}$ & - & $-6.89 \times 10^{-2}$ \\
\hline
\end{tabular}

For ply angle $\phi=0 \mathrm{deg}$, the plate has zero angle of attack, and aerodynamic loads are zero if camber is not included. Note that negative cambers means positive lift and the strip tip moves consequently upwards. For non-zero ply angles there is both twist-bending and twist-camber coupling appearing. The latter results in a significant difference 
between both models in vacuum (see Ref. 24 for details), which gets magnified once the aeroelastic effects are incorporated. The aerodynamic loading in the model with camber deformation also includes the corresponding bimoments (third line in Eq. (30)). However, they are much smaller that the forces created by the piezoactuator and do not create any significant additional deformation in the camber bending degree of freedom.

\section{High-aspect-ratio wing}

The effect of camber bending in the static aeroelastic equilibrium of a very-flexible high-aspect-ratio wing is studied next. The wing model has a constant NACA4406 aerofoil and its chord and span are $2 b=0.5 \mathrm{~m}$ and $L=5 \mathrm{~m}$, respectively. The wing structure is made of a constant cross section with $2.5-\mathrm{mm}$ skin thickness and a spar of the same thickness located at $30 \%$ of the chord. Numerical results were obtained at 2-deg angle of attack at a flight speed of $40 \mathrm{~m} / \mathrm{s}$ and sea level conditions. Camber bending deformations were included by the finite-section mode of Eq. (31). Note that in this case, the airfoil midline has a significant initial camber, whose contribution is always accounted for in the approximation to the airfoil deformations, Eq. (13). Figure 11 compares the vertical displacement, twist rotation and camber deformation (as defined in Figure 4) along the half-chord line of the slender wing corresponding to two 1-D structural models: the "classical" geometrically-nonlinear beam description (extension-twist-bending degrees of freedom), and the present model with finite-section mode to include camber deformations. Finally, Figure 12 compares the 3-D deformed wing in both cases. As it can be observed from Figure 11, camber deformations in the equilibrium position is larger towards the root of the wing and reaches a maximum of about $3 \%$ of the wing chord. This additional camber has the effect of creating a small reduction in both the elastic twist deformations and vertical displacement of the wing.
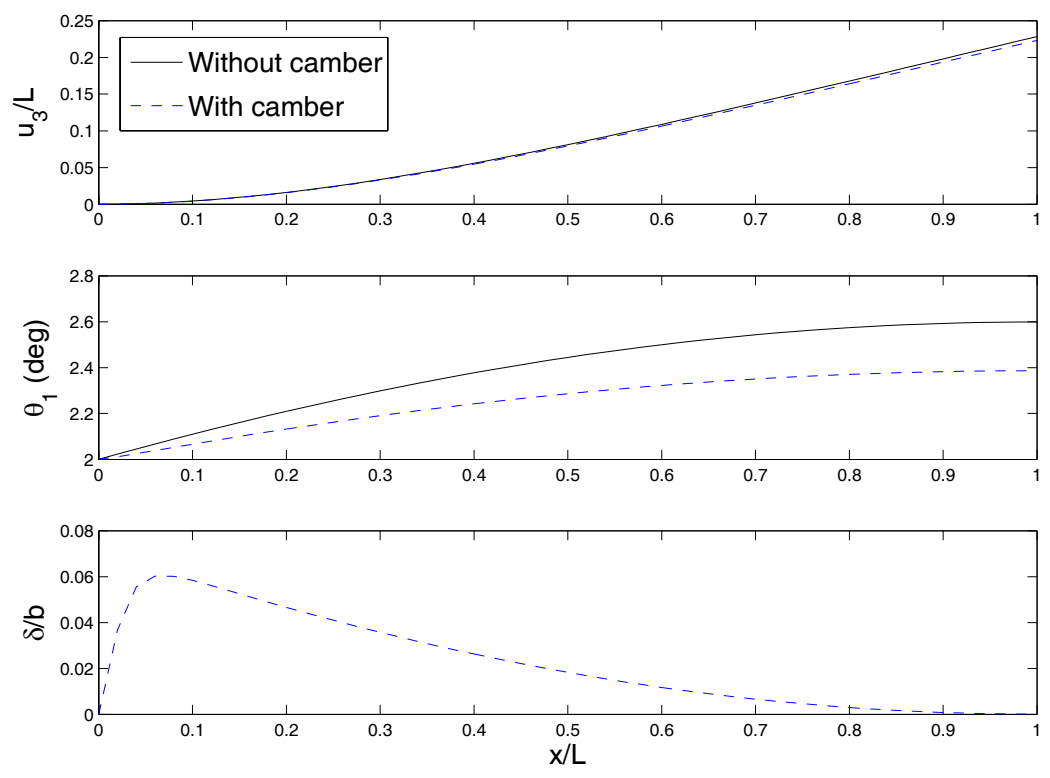

Figure 11. Vertical displacement, twist and camber deformation along the wing midchord $(U=40 \mathrm{~m} / \mathrm{s})$ 


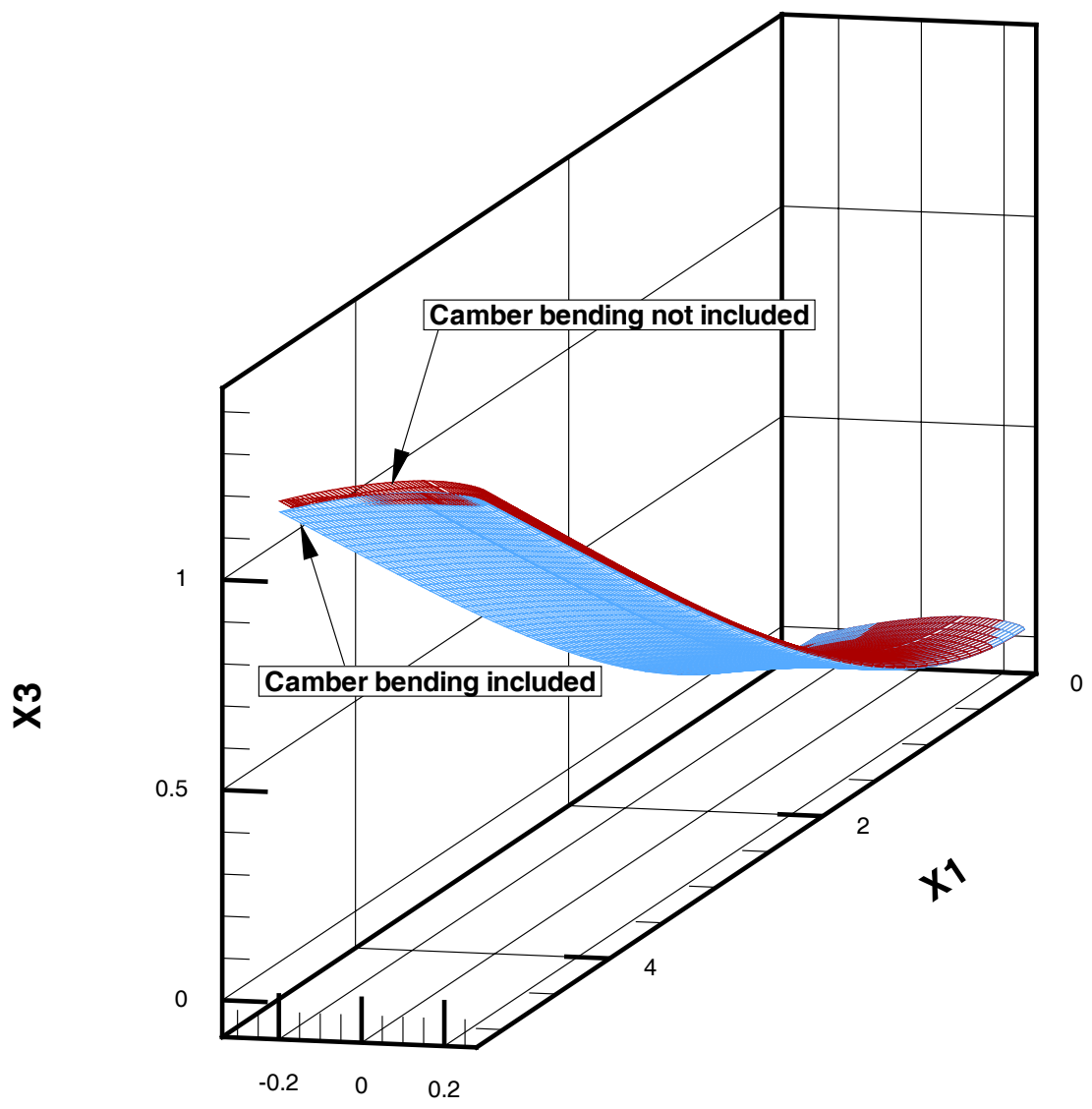

Figure 12. Statically-deformed wing with (blue) and without (red) camber bending $(\mathrm{U}=40 \mathrm{~m} / \mathrm{s})$

\section{Concluding Remarks}

A methodology for the 1-D modeling of wings with deforming airfoils has been introduced. Airfoil deformations are included in both the structural and the aerodynamic model: a Ritz (finite-section) expansion includes cross-sectional structural deformations, while a Glauert expansion accounts for deformations of the airfoil camberline. Both expansions have been integrated in a single methodology and together provide a simple alternative to more complex 2-D and 3-D models for preliminary active aeroelastic analysis of high-aspect-ratio wings and rotorblades with adaptive airfoils. A particularly important situation has been further investigated in this work, corresponding to the analysis of camber deformations of the wing airfoils. For moderately-thin plates, it was observed that the compliance of the structure in that degree of freedom is typically not enough for the aerodynamic loads to create any observable changes in the aeroelastic equilibrium. A more noticeable effect, although still quite small, was observed on a very flexible 5-m-long wing model. However, the selected wing model did not include the effect of ribs, which would in practice prevent the airfoil deformations.

A more interesting case, that was also investigated here, is that of airfoils with integral actuation, in which an internal mechanism generates the shape change. In particular, changes of airfoil camber have been studied on thin plates, both due to general applied bimoments, and from surface-mounted piezoelectric actuators. Results in the 
paper show the effectiveness of actively modifying the airfoil camber to create a desired aeroelastic response of the wing. Such actuation is able to produce instantaneous lift increments which have a much smaller drag penalty than flap deflections. Studies in this work show that several important aspects of camber-type deformation can be assessed through the proposed low-order aeroelastic formulation, but a thorough assessment of the phenomenon with standard 2-D and 3-D aeroelastic analysis tool still needs to be carried out to quantify those improvements with respect to higher-fidelity approximations. Future work will address this issue, as well as the investigation of the usability of the proposed scheme in wing and rotor blade aeroelastic control applications.

\section{Acknowledgements}

This work was supported in part by the Georgia Tech/University of Michigan/Washington University Vertical Lift Rotorcraft Center of Excellence (VLRCOE) funded by the U.S. Army. Dr. Michael Rutkowski is the technical monitor.

\section{References}

${ }^{1}$ Cesnik CES, Shin S, Wilbur ML (2001). "Dynamic response of active twist rotor blades." Smart Materials and Structures 10 (1) p 62-76.

2 Santer M, Pellegrino S (2007). “Topology Optimization of Adaptive Compliant Aircraft Wing Leading Edge." Proceedings of the 48th AIAA/ASME/ASCE/AHS/ASC Structures, Structural Dynamics, and Materials Conference, Waikiki, Hawaii, USA, April 2007. AIAA Paper 2007-1714.

${ }^{3}$ Campanile LF, Anders S (2005). "Aerodynamic and Aeroelastic Amplification in Adaptive Belt-Rib Airfoils." Aerospace Science and Technology 9 (1) p 55-63.

${ }^{4}$ Bernhard APF, Chopra I (2001). "Analysis of a bending-torsion coupled actuator for a smart rotor with active blade tips." Smart Materials and Structures 10 (1) p 35-52.

5 Parker HF (1920). "The Parker Variable Camber Wing." NACA Report No 77.

${ }^{6}$ Gandhi F, Frecker M, Nissly A (2008). "Design Optimization of a Controllable Camber Rotor Airfoil." AIAA Journal 46 (1) $\mathrm{p}$ 142-153.

7 Panza JL, Suciu EO (2005). "A Closer Look at the Elastic Streamwise Camber Deformation of Swept and Unswept Wings." IFASD International Forum of Aeroelasticity and Structural Dynamics, Munich, Germany July 2005

${ }^{8}$ Palacios R, Cesnik CES (2005). "On the application of asymptotic reduction methods of slender structures to fluidstructure interaction problems.” In Bathe K.J. (ed), Third M.I.T. Conference in Computational Fluid and Solid Mechanics, Cambridge, Massachusetts, USA, p 612-616.

${ }^{9}$ Sears WR (1941). "Some Aspects of Non-Stationary Airfoil Theory and Its Practical Application." Journal of the Aeronautical Sciences 8 (3) p 104-108.

${ }^{10}$ Theodorsen T (1935). "General theory of aerodynamic instability and the mechanism of flutter." NACA Report No. 496.

${ }^{11}$ Wu TY (1961). “Swimming of a wawing plate." Journal of Fluid Mechanics 10 (3) p 321-324.

${ }^{12} \mathrm{Wu}$ TY (1971). "Hydromechanics of swimming propulsion. Part 1. Swimming of a two-dimensional flexible plate at variable forward speeds in an inviscid fluid.” Journal of Fluid Mechanics 46 (2) p 337-355.

${ }^{13}$ Peters, David A. and Johnson, Mark J., "Finite-State Airloads for Deformable Airfoils on Fixed and Rotating Wings," Aeroelasticity and Fluid Structure Interaction Problems, Proceedings of the ASME Winter Annual Meeting, Chicago, Illinois, USA, AD-Vol. 44, pp. 1-28, November 6-11, 1994.

${ }^{14}$ Peters DA, Hsieh MA, Torrero A (2006). "A State-Space Airloads Theory for Flexible Airfoils." Proceedings of the 62 ${ }^{\text {nd }}$ Annual Forum of the American Helicopter Society, Phoenix, Arizona, USA, May 2006. 
${ }^{15}$ Palacios R, Cesnik CES (2008), “A Geometrically-Nonlinear Theory of Composite Beams with Deformable Cross Sections." AIAA Journal 46 (2) p 439-450.

${ }^{16}$ Cesnik CES, Hodges DH (1997), "VABS: A New Concept for Composite Rotor Blade Cross-Sectional Modeling." Journal of the American Helicopter Society 42 (1), p 27-38.

${ }^{17}$ Palacios R, Cesnik CES (2005), "Cross-Sectional Analysis of Non-Homogeneous Anisotropic Active Slender Structures." AIAA Journal 43 (12) p 2624-2638.

${ }^{18}$ Hodges DH (1990). “A Mixed Variational Formulation Based on Exact Intrinsic Equations for Dynamics of Moving Beams.” International Journal of Solids and Structures 26 (11) p 1253-1273.

${ }^{19}$ Yu W, Hodges DH, Volovoi VV, Cesnik CES (2002). "On Timoshenko-Like Modeling of Initially Curved and Twisted Composite Beams.” International Journal of Solids and Structures 39 (19) p 5101-5121.

${ }^{20}$ Danielson DA, Hodges DH (1987). "Nonlinear Beam Kinematics by Decomposition of the Rotation Tensor." ASME Journal of Applied Mechanics 54 (2) p 258-262.

${ }^{21}$ Cesnik CE.S, Hodges DH (1997). "VABS: A New Concept for Composite Rotor Blade Cross-Sectional Modeling." Journal of the American Helicopter Society 42 (1) p 27-38.

${ }^{22}$ Abramowitz M., Stegun I.A., Handbook of Mathematical Functions, $9^{\text {th }}$ Edition. Dover, Mineola, NY, 1972.

${ }^{23}$ Peters DA, Karunamoorthy S, and Cao W (1995). "Finite State Induced Flow Models Part I: Two-Dimensional Thin Airfoil." Journal of Aircraft 32 (2) p 313-322.

${ }^{24}$ Palacios R, Cesnik CES (2008). "On the one-dimensional modeling of camber bending deformations in active anisotropic slender structures.” International Journal of Solids and Structures 45 (7-8) p 2097-2116. 